\title{
Surviving in a Competitive Spatial Market: The Threshold Capture Model*
}

\author{
Daniel Serra ${ }^{\dagger}$ \\ Charles ReVelle \\ and \\ Ken Rosing $\S$
}

February 2, 1999

\begin{abstract}
Most facility location decision models ignore the fact that for a facility to survive it needs a minimum demand level to cover costs. In this paper we present a decision model for a firm that wishes to enter a spatial market where there are several competitors already located. This market is such that for each outlet there is a demand threshold level that has to be achieved in order to survive. The firm wishes to know where to locate its outlets so as to maximize its market share taking into account the threshold level. It may happen that due to this new entrance, some competitors will not be able to meet the threshold and therefore will disappear. A formulation is presented together with a heuristic solution method and computational experience.
\end{abstract}

jel: C61,R12,R53

keywords: discrete facility location, threshold, competitive location.

\footnotetext{
*This research has been partially financed by DCYGIT grant PB95-0980, Ministry of Education (Spain). Please do not quote without the author's permission.

${ }^{\dagger}$ Dept. of Economics and Business, Universitat Pompeu Fabra

$\ddagger$ Dept. of Geography and Environmental Engineering, The Johns Hopkins University

$\S$ Dept. of Applied Economics, Erasmus University
} 


\section{Introduction}

While the concept of market threshold has been extensively used in central place theory and in conjectural location theory, its use in facility location decision models is quite scarce compared to the large amount of existing literature in this research area. This scarcity is particularly true in retail location modelling, where threshold levels may be a substantial part of success in the retail activity. It is widely recognized in the retail literature that "there is some minimum size of a market below which a place will be unable to supply a central good... and is here termed the threshold sales level for the provision of that good from the center" (Berry and Garrison, 1958, p.111 as cited by Shonkwiler and Harris, 1996). In fact, several authors have recognized that there is a demand entry threshold which is a measure of the market size to support a given number of firms. Or, in other words, retailfirm entry thresholds are largely determined by local populations (Bresnahan and Reiss 1991, Shonkwiler and Harris, 1996).

Another way of looking at this issue is by studying the concept of market saturation. It may happen that a geographical market may become saturated. This notion of saturation of a retail market implies that additional provision would prove "unsustainable for a retail organization in the context of available consumer demand". In other words, the market size has attained its threshold level, and the entry of any new retailer is no longer profitable. (Jonatan Reynolds, cited in IGD, page 245)

The importance of market threshold is demonstrated by the fact that most retail franchisers give their franchisees some kind of territorial exclusivity, that other outlets of the same chain will not be opened within a given distance from an existing one on the region. Even if in the contract between the franchiser and the franchisee the sale territory exclusiveness is not recognized, several courts have ruled that all contracts have an implied covenant of good 
faith and fair dealing: "...Although not entitled to an exclusive territory, the franchisee was still entitled to expect that the franchisor would not act to destroy the right of the franchisee to enjoy the fruits of the contract" (Vylene Enterprises, Inc. vs Naugles, Inc., 1996 WL 420412, 96 Daily Journal D.A.R. 9087).

In this paper we present a decision model for a retail firm (from now on Firm A) that wishes to enter a spatial market where there are several outlets already located. This market is such that for each outlet of the existing or the entering firm there is a demand threshold level that has to be achieved in order for the outlet to survive. The firm wishes to know where to locate its outlets so as to maximize its total market share (or in other words, market capture) taking into account the threshold level. The rule for capture of a demand area is that capture occurs if the demand area is made closer to an entering outlet than its previously closest existing outlet. Otherwise, all market capture will go to the closest existing outlet. It may happen that due to this new entrance, some competitors' outlets will not be able to enjoy the threshold level of demand and therefore will disappear. We will assume that the existing outlets belong to only one competitor firm (Firm B), without loss of generality.

Current and Storbeck (1994) formulated a multiobjective model that selects franchise locations and identifies individual franchise market areas. Constraints in their formulation guarantee that all franchise locations are assigned at least a minimal threshold market area with sufficient demand to ensure economic survival. A similar model was formulated by Ghosh and Craig (1991). They considered the impact of a new outlet on form and brand demand, and on competition. They developed a network optimization model to evaluate potential sites for their impact on system revenues and on existing outlets.

Threshold constraints have also been included in covering models by Bal- 
akrishnan and Storbeck (1991). In their model (McTHRESH) they addressed the issue of locating a given number of outlets so that market coverage is maximized within some pre-determined range, and the required threshold level of demands are maintained for all sites. Later, they used the McTHRESH model as a site-generation technique, developing network configurations where each configuration was optimal under different parametric conditions. Once these scenarios were generated, they used additional criteria to select the most efficient among them.

When new services are sited in a spatial market where there are other servers already operating, it may happen that the threshold requirement of the existing servers may not be achieved due to the reduction in their catchment area caused by the new entry. For example, when locating new outlets in a competitive environment where there are other competitor outlets already operating, the market share of existing outlets will be reduced and some of these outlets may go out of business due to market saturation. Conversely, not every new outlet trial will result in a viable outcome due to the requirement for capture of a minimal demand level. A similar situation may occur when locating public facilities in a region where some facilities already exist. This new entrance may transform some existing facilities that were efficient into facilities that are no longer efficient due to the reduced size of their catchment area.

The paper is organized as follows. In section 2, a decision model is presented to locate facilities with the threshold requirement in the presence of other outlets. In section 3, a meta-heuristic based on heuristic concentration (Rosing 1997, Rosing and ReVelle 1997) is developed. Section 4 presents some computational experience on different sized networks. In section 5 an example is presented on a 55-node network. Finally, Section 6 concludes the paper, summarizes the investigation and draws some conclusion on the model and solution methods. 


\section{The Model}

The model space is represented by a discrete network, where consumers are located at specific points, such as vertices of the network. Potential locations for the services are also pre-specified.

Lets define $a_{i}$ as a measure of demand to be served at node $i$. We assume that demand is totally inelastic, that is, that consumers decide to buy a given amount of the good regardless of its price. The good sold is homogeneous and consumers will purchase it at the closest outlet. The threshold level can be defined as the minimum expected amount of demand necessary to cover costs, or as the minimum number of customers required. Price is set exogenously, and consumers bear transportation costs. The formulation of the model is as follows:

$$
\max Z=\sum_{i \in I} \sum_{j \in J} \alpha_{i j} x_{i j}
$$

Subject to:

$$
\begin{array}{rlrl}
\sum_{j \in J} x_{i j} & =1 & & i \in I \quad(1) \\
x_{i j} \leq w_{j} & i \in I, & j \in J \quad(2) \\
\sum_{i \in I} a_{i} x_{i j} \geq C w_{j} & j \in J \quad(3) \\
\sum_{j \in J} w_{j} & =p & & \\
x_{i j}, w_{j} & \in\{0,1\} & i \in I, \quad j \in J &
\end{array}
$$

where: 


$$
\begin{aligned}
i, I & =\text { index and set of demand areas } \\
j, J & =\text { index and set of potential locations } \\
b_{i} & =\text { index of the closest existing outlet to node } i \\
a_{i} & =\text { Demand at node } i \\
d_{i j} & =\text { distance between node } i \text { and node } j \\
C & =\text { Minimum number of customers required for an outlet to survive } \\
p & =\text { number of facilities to locate } \\
x_{i j} & =1, \text { if node } i \text { is served by a facility at node } j ; 0 \text {, otherwise } \\
w_{j} & =1, \text { if an outlet is open at node } j, 0 \text { otherwise } \\
\alpha_{i j} & =a_{i} \text { if } d_{i j}<d_{i b_{i}} ; 0 \text {, otherwise }
\end{aligned}
$$

This model is similar to the Maximum Capture Model (MAXCAP), formulated by ReVelle (1986). The MAXCAP model seeks the location of a given number of facilities in a discrete network so as to maximize market share captured. The basic difference between the two models is the threshold constraint (3) which does not exist in the MAXCAP model.

The variable $x_{i j}$ assigns customers to facilities. Since customers make purchases at their closest facility, this variable is binary. Therefore, consumers are assigned to only one facility. Constraint group (1) forces this to happen. Since each demand node $i$ is assigned to only one facility, the sum of $x_{i j}$ with respect to $j$ has to be equal to 1 . But to allow $x_{i j}$ to be one, at least one facility has to be open at some node $j$. The second group of constraints allows $x_{i j}$ to be one only if $w_{j} \geq 1$. If $w_{j}=0$, assignment will be forbidden. The third group of constraints allows a facility to open at $j$ only if the total demand assigned to node $j$ is at least equal to the threshold level $C$. The last constraint limits the number of entering facilities to $p$ in number.

The objective is to maximize the population captured by the entering outlets. The parameter $\alpha_{i j}$ is equal to $a_{i}$ if the distance from demand node $i$ to the potential facility node $j$ is less than the distance from $i$ to the closest existing outlet $b_{i}$. If the distance is equal or greater, the demand remains with the closest existing outlet.

Observe that the number of facilities to locate is fixed. The model can be 
easily modified if the objective is to maximize profits by using the following objective:

$$
\max Z=\sum_{i \in I} \sum_{j \in J} \alpha_{i j} x_{i j}-\sum_{j \in J} f_{j} w_{j}
$$

where $\alpha_{i j}$ is equal to $a_{i}$ if $d_{i j}<d_{i b_{i}}$, where $a_{i}$ is now interpreted as the expected revenues obtained from node $i$; and equal to 0 otherwise; and $f_{j}$ is the cost of opening an outlet at node $j$.

The threshold model presented here can be solved using linear programming and branch and bound when necessary $(\mathrm{LP}+\mathrm{BB})$ for relatively small networks. But, still this method can become burdensome as the network size increases. First, the method can be burdensome because the number of variables and constraints can increase dramatically, even for medium-size networks. For example, if the number of nodes is equal to 50, the problem will have 2500 binary variables $x_{i j}$ and 2601 constraints. If the number of nodes is 100, the number of binary variables jumps to 10000 variables. There are several methods to reduce the number of variables and constraints, but the problem may still be intractable (see Rosing et al. 1979)). Second, the threshold constraint creates an additional problem in finding integer solutions since the specified parameters are no longer equal to 0 or 1 . This implies that most likely the number of branches can increase dramatically (see ReVelle (1993) on Integer Friendly Programming). Even if a solution of the problem can be obtained using $\mathrm{LP}+\mathrm{BB}$, the final assignment of nodes to facilities may not be correct, since we have assumed that consumers go to the closest facility, and some nodes may assign to a facility that is not closest, a situation that can occur in capacitated problems.

Another problem arises when the final solution is obtained from the model. It may happen that by opening Firm A's new outlets some of the demand that was originally assigned to Firm B's existing outlets is reassigned to the new ones. Those Firm B outlets unable to meet the individual 
threshold requirement will collapse since they become threshold deficient. If threshold deficiency occurs, the demand of the collapsed outlet is reassigned to the remaining outlets including both new and existing servers. But, what happens if two existing outlets do not meet the threshold requirements in such a way that, if one of them dies, the other one could survive due to the demand re-allocation? Should both of them disappear, or only one? It is necessary to establish the following initial set of survival rules:

\section{Set of rules 1:}

1. The entering firm always locates in nodes where the threshold requirement is met

2. Once the entering firm has located its outlets, demand re-allocation to the closest outlet is done. If there are two or more existing competitor outlets that do not meet the threshold requirement, the one with the lowest market share "dies" first and its allocated demand is re-assigned to its closest outlet whether existing or new. The procedure is repeated for the outlets that still do not meet the threshold constraint. The procedure stops when all the remaining outlets satisfy the threshold requirement.

Therefore, the set of rules 1 can be applied once locations are found using linear programming + branch and bound, and they are used to obtain the final capture by both firms. The implication is that the solution of the integer program may provide a very good answer to the problem, but that demand re-shuffling brought about by outlet collapse could still improve the value

of the objective for Firm A, suggesting that the objective value from the optimization was not quite calculated correctly.

In the following section a heuristic method is presented to solve the problem. The heuristic locates Firm A servers with outlet death of Firm B servers 
as a possible consequence of Firm A's chosen sites. That is, the heuristic calculates demand capture by Firm A with outlet death included in the equation.

\section{A Heuristic Concentration Algorithm to Solve the Model}

Heuristic methods for specific capacitated location problems have been studied by Pirkul and Schilling (1991), Current and Storbeck (1988) (capacitated covering models) and by Cornuejols et al. (1991) (capacitated plant location problems) among others.

In this paper a heuristic concentration (HC) algorithm is presented. Heuristic concentration methods have been proposed by Rosing (1997) and Rosing and ReVelle (1997). In essence, the HC method has two phases. In the first phase, multiple runs of an interchange heuristic or other heuristic are executed from different initial solutions. In the second phase, a heuristic or exact procedure is used to obtain a solution from a concentrated set of potential locations generated by the first phase. In order to solve the threshold capture model, we use in both phases of the HC heuristic the well-known Teitz and Bart (1968) one-opt heuristic, improved by Densham and Rushton (1992).

A formal description of the procedure follows.

PhASE I

1. Find $p$ random initial locations for Firm A outlets.

2. Find the demand served by each Firm A outlet. If the threshold requirement is not met by any of the $p$ outlets, set the objective to zero and go to step 3. Otherwise, apply set of rules 1 to see if there is outlet "death". Compute the final capture obtained by Firm A once all demand reassignment has taken place. 
3. Choose the first Firm A outlet from a list of its outlets and trade its location to an empty node within the set of potential locations.

4. Find again the demand served by each Firm A outlet. If the threshold requirement is not met, set the objective to zero and go to step 5. Otherwise, Apply set of rules 1 to see if there is outlet "death". Compute the final capture obtained by Firm A. If this objective has improved, store the new locational solution. If not, restore the old solution.

5. Repeat steps 4 and 5 until all potential empty locations have been evaluated one at a time for each outlet.

6. If Firm A has improved its market share to a value greater than in step 2 , go to step 3 and restart the procedure.

7. When no improvement is achieved on a complete set of one-at-a-time trades, store final solution.

8. Goto step 1 until a fixed number of iterations of Phase 1 is met.

\section{PHASE II}

- Use all final locations obtained from all starting solutions or use the final locations from the best $k$ out of the multiple starting solutions in Phase I to form the new reduced set of potential locations. Repeat Phase 1 for only one iteration.

\section{Computational Experience}

The algorithm has been applied to several randomly generated networks having the number of nodes $n$ equal to 20,35 and 50. For each $n$ three different threshold levels $C$ were set using the following formula: $C=\beta[p o p /(p+q)]$ where $p o p$ is the total amount of demand to be served, $p$ and $q$ are the number of entering outlets and the number of outlets that are already operating 
respectively, and $\beta$ is a threshold factor that was set to $0.3,0.5$ and 0.7 . As $\beta$ is increased, the higher the threshold factor is, and therefore more outlets' death may happen.

For each $n$ and each $C$, three different number of outlets were used so that $p=q=2,3,4$. Finally, for each $n$, each $C$ and each $p$ and $q$ ten networks were generated randomly. Therefore, a total of 270 networks were generated. All the generated nodes were both demand centers and potential locations. The demand in each node was randomly generated between 50 and 100 using a uniform distribution.

For each generated network, the locations of the $q$ existing outlets were found using the Teitz and Bart heuristic with a p-median objective. The number of iterations in the first phase of the heuristic concentration was arbitrarily set to $4 * n$. Optimal solutions were also obtained using complete enumeration. The heuristic was programmed in FORTRAN77 and executed with a Pentium PC 75 with $24 \mathrm{mb}$ of RAM. Results are shown in Table 1.

(Table 1 about here)

The number of non optimal solutions is presented in the column labeled NON OPT. If at least a non-optimal solution is found among the ten runs, the average and maximum deviation from optimality are presented in the two next columns respectively. The average market capture obtained by the entering outlets is shown in the next column. Finally, average execution time in seconds is presented in the last column.

Only the solutions of 10 out of 270 runs were non-optimal based on our comparison with complete enumeration. In general the average deviation from optimality did not exceed $5 \%$ except when $n=50, \beta=0.7$ and $n p=3$, where 2 non-optimal solutions were found and in one case the deviation from optimality was equal to $14.1 \%$. In most cases the final market capture by the entering outlets was over $50 \%$, even though the existing outlets were well 
positioned and, in case of ties, the existing outlets take all the market.

Finally, the average computing time of the heuristic increased with the number of nodes and the number of outlets, as expected. For example, for $n=50, \beta=0.7$ and $p=4$, the first phase of the heuristic executed 200 runs of the Teitz and Bart heuristic to generate the concentration set to be used in the second phase of the heuristic. In this case, on average the heuristic took 1 minute to execute.

\section{An Example}

The problem was also tested in the well-known Swain's (1974) 55-nodes network (see appendix 1). The demand at each node is indicated in the appendix. The total amount of demand to be captured is equal to 3575 . Distances were computed using a shortest path algorithm. The model was solved to optimality by using complete enumeration.

In this example, 4 outlets are already operating in the market by firm B, and they are located at nodes 1, 16, 29 and 41 (see figure 1). The threshold factor $\beta$ was set to 0.80 , giving a threshold level equal to 357.5. Now, a new firm, Firm A, enters with four new outlets. Due to the entrance of the new outlets, some of the existing outlets were not able to survive after applying the set of rules 1. This situation can be observed in Figure 1. The first picture in Figure 1 shows the capture obtained by each Firm B's existing outlet before Firm A enters. Picture 2 shows the new locational situation after Firm A enters with four outlets. Due to this entrance, the market area of the existing outlets is reduced significantly. Table 2 shows the final capture of each firm's outlets. While all Firm A outlets meet the threshold level, some of Firm B outlets are below it. Therefore, the set of rules 1 has to be used.

(Figure 1 about here) (Table 2 about here) 
In a first step, firm B's outlet in node 41 is the outlet with the lowest market capture and it is not able to survive since its capture is below the threshold level. Therefore, this outlet closes and its demand is reassigned among the other outlets. This situation is depicted in picture 3. Now, the outlet in node 29 is the one that has the lowest market capture, and again it does not meet the threshold requirement. Once again, it does not survive and its demand is reassigned among the other outlets (picture 4). Finally, the same situation occurs with Firm B's outlet located in node 16 and therefore it disapears from the market. The final outcome is depicted in picture 5, where all the outlets meet the threshold level. Firm A's final capture represents $73 \%$ of the market share, while Firm B has only one outlet left with a total capture of the remaining $27 \%$.

The model presented so far assumes that Firm A will always locate its outlets in nodes where the threshold level is met. But what happens if an entering outlet is located so that, even though once located it does not meet the threshold level, but after the outlet "death" of some of the existing outlets its threshold level is met? In other words, suppose that Firm A locates an outlet where the threshold level is not met by, lets say, 5\%, but its entry causes an existing outlet to deviate from the threshold level by $20 \%$. If the existing outlet disappears, the entering outlet meets the threshold constraint. Therefore, Firm A may take the risk of positioning its outlet there, even though the threshold requirement is not met, since it will cause a weaker outlet to "die". The following set of rules may be used:

\section{Set of rules 2:}

1. The entering firm locates $p$ outlets in nodes without checking if the threshold requirement is met

2. Once the entering firm has located its outlets, demand re-allocation to the closest outlet is done. If there are two or more existing and 
entering outlets that do not meet the threshold requirement, the one with the lowest market capture "dies" first and its allocated demand is re-assigned to its closest outlet. The procedure is repeated for the outlets that still do not meet the threshold constraint. The procedure stops when all the remaining outlets satisfy the threshold requirement.

Observe that if the second set of rules is applied some of the $p$ Firm A's outlets may not survive and will disappear too. The heuristic presented may be adapted to this new set of rules.

In order to test which set of rules favors the entering firm, additional computational experience was performed on the Swain network as follows: the number of outlets $p$ and $q$ for each firn was set to 2, 3 and 4 (where $p=q)$. For each one of them, the $\beta$ factor was set to $0.2,0.4$ and 0.6. Then, for each $p$ and $\beta, 20$ different scenarios were randomly generated by allowing the population at each node to vary between 30 and 100, and with different outlet locations of the competitor. For each run, both sets of rules were used. That is, a total of 320 different runs were performed. In most of the runs $(93,8 \%)$ both rules achieved the same results. In the remaining $6.2 \%$, the set of rules 2 gave better results for the entering firm. No results were found were the set of rules 1 would achieve a better market capture. This was expected, since the algorithm related to set of rules 2 allows to visit more potential locations, since all nodes are potential candidates to receive a facility, regardless of the inicial threshold level. The difference in these runs is shown in Table 3 together with the initial and final number of outlets for each firm.

The second set of rules was used in the test network. The threshold level is set to 357.5 as before. Again, Firm B outlets are located in nodes 1, 16, 29 and 41. Firm A enters with four outlets. These are sited at nodes 2, 13, 23 and 32. Results are presented in Table 4 and Figure 2. First, observe that Firm B's outlet located at 1, 16 and 29 and Firm A's outlet at 32 do not 
meet the threshold level. The second set of rules is applied. Firm B's outlet at node 1 is the one with the lowest market capture and therefore disappears (picture 2 in Figure 2). Its demand is re-assigned to the remaining outlets. After re-assignment, Firm A's outlet at 32 and Firm B's outlet at 16 and 29 still do not meet the threshold level. This time, Firm B's outlet at 16 is the outlet with the lowest market capture and therefore its closed and its demand is re-allocated to the other servers (picture 3 in Figure 2). Now, due to this re-allocation, only Firm B's outlet at node 29 remains below the threshold level (picture 4). Its closure leads to the final situation depicted in picture 5 .

By using in the algorithm the set of rules 2 Firm A achieved a better capture than by using set of rules 1, since in each iteration Firm A outlets were allowed to locate in nodes where the threshold was not met before applying the set of rules. Recall that when using the set of rules 1 in this example the model was solved to optimality.

((Figure 2 about here) (Table 3 about here)

\section{Conclusions}

In this paper a new location model has been presented to study the issue of minimum threshold requirements to survive in a given spatial setting. The model is very relevant in both public and private sector settings. In the public sector, when planning the expansion of service delivery where there are merit goods involved, such as, for example, post offices, pharmacies or tax offices, the threshold level may determine the minimum service level to make the outlet effective. In the private sector, the opening of new outlets in the presence of competition requires placement such that these outlets will survive by having enough demand. Therefore, in both cases the threshold constraint can be considered as a search for efficiency, in the sense that each 
facility needs a given threshold demand/revenue/area below which it is not viable. A concentration algorithm has been developed to solve the problem. 
FIGURE 1

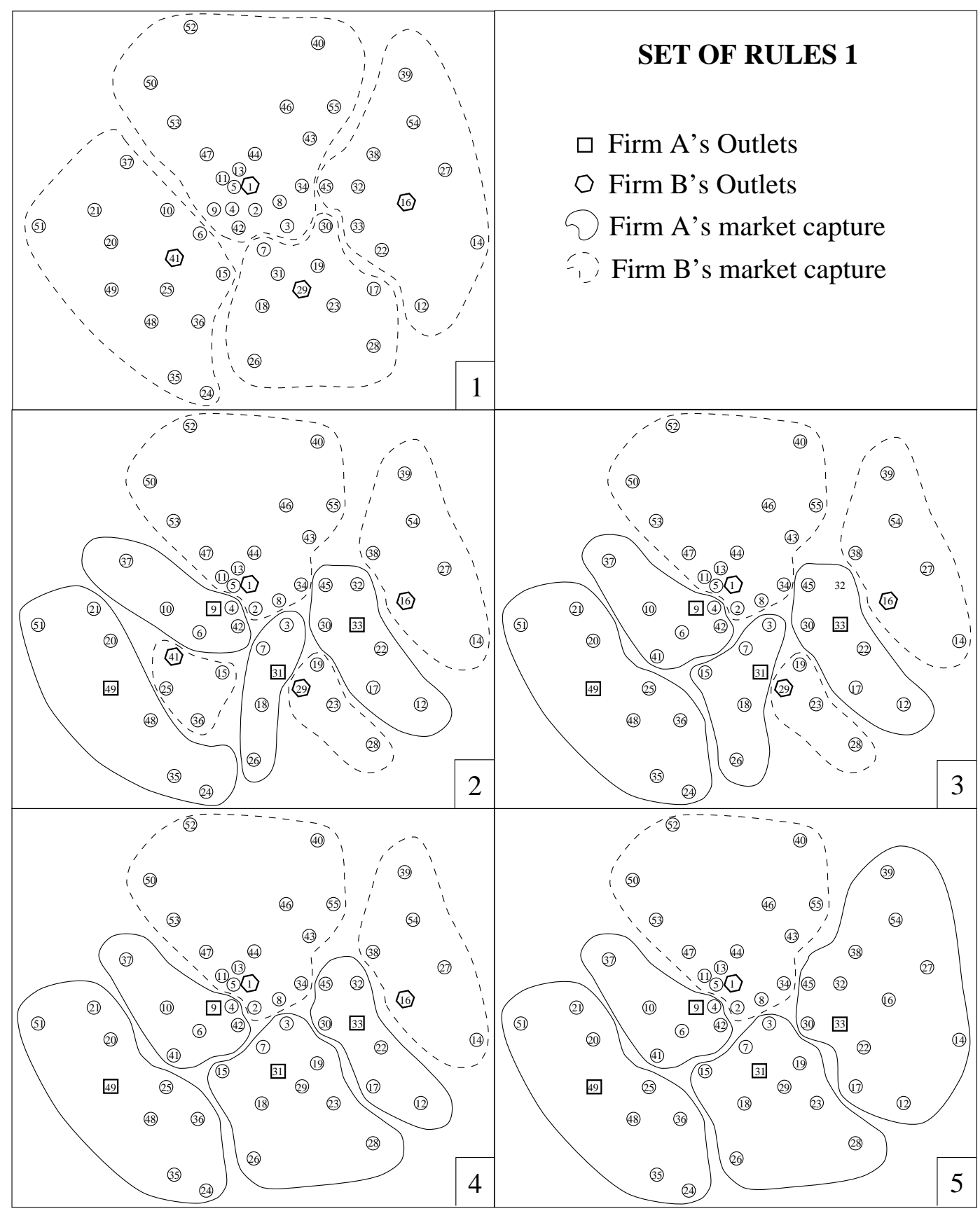


FIGURE 2

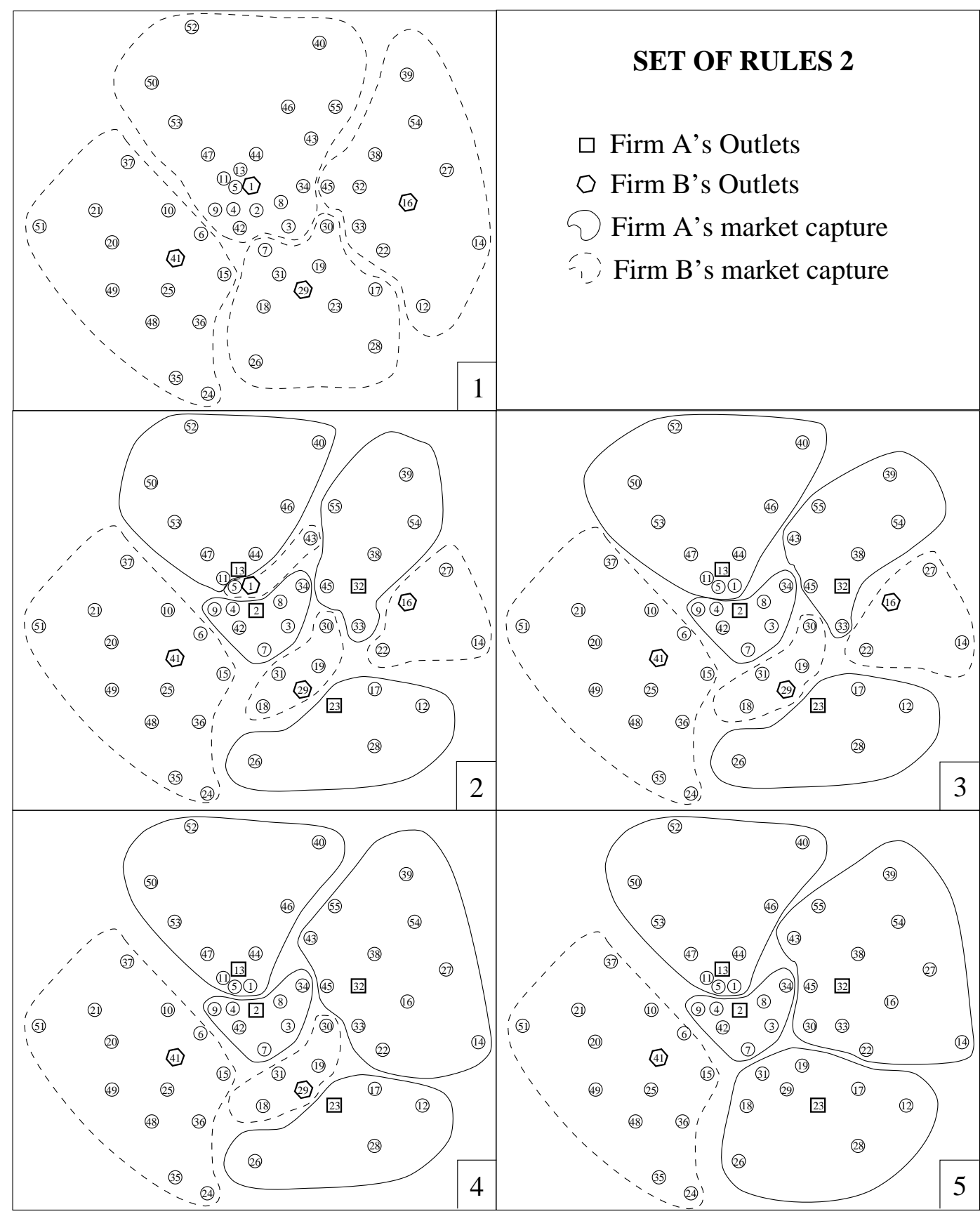




\section{References}

Balakrishnan, P. and Storbeck, J. (1991). Mctresh: Modeling maximum coverage with threshold constraints. Environment \& Planning B - Planning and Design, 18(4):459-472.

Berry, B. J. and Garrison, W. (1958). Recent developments of central place theory. Papers and Proceedings of the Regional Science Association, 4:107-120.

Bresnahan, T. and Reiss, P. (1991). Entry and competition in concentrated markets. Journal of Political Economy, 99:977-1009.

Cornuejols, G., Sridharan, R., and Thizy, J. (1991). A comparison of heuristics and relaxations for the capacitated plant location problem. European Journal of Operational Research, 50:280-297.

Current, J. and Storbeck, J. (1988). Capacitated covering models. Environment and Planning B, 15:153-164.

Current, J. and Storbeck, J. (1994). A multiobjective approach to design franchise outlet networks. Journal of Operational Research Society, $45: 71-81$.

Densham, P. and Rushton, G. (1992). Strategies for solving large locationallocation problems by heuristic methods. Environment and Planning A, 24:289-304.

Ghosh, A. and Craig, S. (1991). FRANSYS: A franchise location model. Journal of Retailing, 67:212-234.

IGD (1993). Superstores Trading Profile 92. Institute of Grocery Distribution, Letchmore Heath, Watford WD2 8DQ. 
Pirkul, H. and Schilling, D. (1991). The maximal covering location problem with capacities on total workload. Management Science, 37(2):233-248.

ReVelle, C. (1986). The maximum capture or sphere of influence problem: Hotelling revisited on a network. Journal of Regional Science, 26(2):343357.

ReVelle, C. (1993). Facility siting and integer-friendly programming. European Journal of Operational Research, 65:147-158.

Rosing, K. (1997). An empirical investigation of the effectiveness of a vertex substitution heuristic. Environment and Planning B, 24:59-67.

Rosing, K. and ReVelle, C. (1997). Heuristic concentration: Two stage solution construction. European Journal of Operational Research, 97:75-86.

Rosing, K., ReVelle, C., and Rosing-Vogelaar, H. (1979). The p-median model and its linear programming relaxation: an approach to large problems. Journal of the Operational Research Society, 30(9):815-823.

Shonkwiler, J. and Harris, T. (1996). Rural retail business thresholds and interdependencies. Journal of Regional Science, 36(4):617-630.

Swain, R. (1974). A parametric decomposition algorithm for the solution of uncapacitated location problems. Management Science, 21:189-198.

Tietz, M. B. and Bart, P. (1968). Heuristic methods for estimating the generalized vertex median of a weighted graph. Operations Research, $16: 955-965$. 


\section{Appendix: 55-node Network demands and co- ordinates}

\begin{tabular}{|c|c|c|c|c|c|c|c|c|c|c|c|}
\hline node & pop & \multicolumn{2}{|c|}{ coord } & node & pop & & & node & pop & \multicolumn{2}{|c|}{ coord } \\
\hline & & $\mathrm{x}$ & $\mathrm{y}$ & & & $\mathrm{x}$ & $\mathrm{y}$ & & & $\mathrm{x}$ & $\mathrm{y}$ \\
\hline 1 & 120 & 32 & 31 & 20 & 77 & 25 & $\overline{14}$ & 39 & 47 & 46 & 51 \\
\hline 2 & 114 & 29 & 32 & 21 & 76 & 29 & 12 & 40 & 44 & 50 & 40 \\
\hline 3 & 110 & 27 & 36 & 22 & 74 & 24 & 48 & 41 & 43 & 23 & 22 \\
\hline 4 & 108 & 29 & 29 & 23 & 72 & 17 & 42 & 42 & 42 & 27 & 30 \\
\hline 5 & 105 & 32 & 29 & 24 & 70 & 6 & 26 & 43 & 41 & 38 & 39 \\
\hline 6 & 103 & 26 & 25 & 25 & 69 & 19 & 21 & 44 & 40 & 36 & 32 \\
\hline 7 & 100 & 24 & 33 & 26 & 69 & 10 & 32 & 45 & 39 & 32 & 41 \\
\hline 8 & 94 & 30 & 35 & 27 & 64 & 34 & 56 & 46 & 37 & 42 & 36 \\
\hline 9 & 91 & 29 & 27 & 28 & 63 & 12 & 47 & 47 & 35 & 36 & 26 \\
\hline 10 & 90 & 29 & 21 & 29 & 62 & 19 & 38 & 48 & 34 & 15 & 19 \\
\hline 11 & 88 & 33 & 28 & 30 & 61 & 27 & 41 & 49 & 33 & 19 & 14 \\
\hline 12 & 87 & 17 & 53 & 31 & 60 & 21 & 35 & 50 & 33 & 45 & 19 \\
\hline 13 & 87 & 34 & 30 & 32 & 58 & 32 & 45 & 51 & 32 & 27 & 5 \\
\hline 14 & 85 & 25 & 60 & 33 & 57 & 27 & 45 & 52 & 26 & 52 & 24 \\
\hline 15 & 83 & 21 & 28 & 34 & 55 & 32 & 38 & 53 & 25 & 40 & 22 \\
\hline 16 & 82 & 30 & 51 & 35 & 54 & 8 & 22 & 54 & 24 & 40 & 52 \\
\hline 17 & 80 & 19 & 47 & 36 & 53 & 15 & 25 & 55 & 21 & 42 & 42 \\
\hline 18 & 79 & 17 & 33 & 37 & 51 & 35 & 16 & & & & \\
\hline 19 & 79 & 22 & 40 & 38 & 49 & 36 & 47 & & & & \\
\hline
\end{tabular}

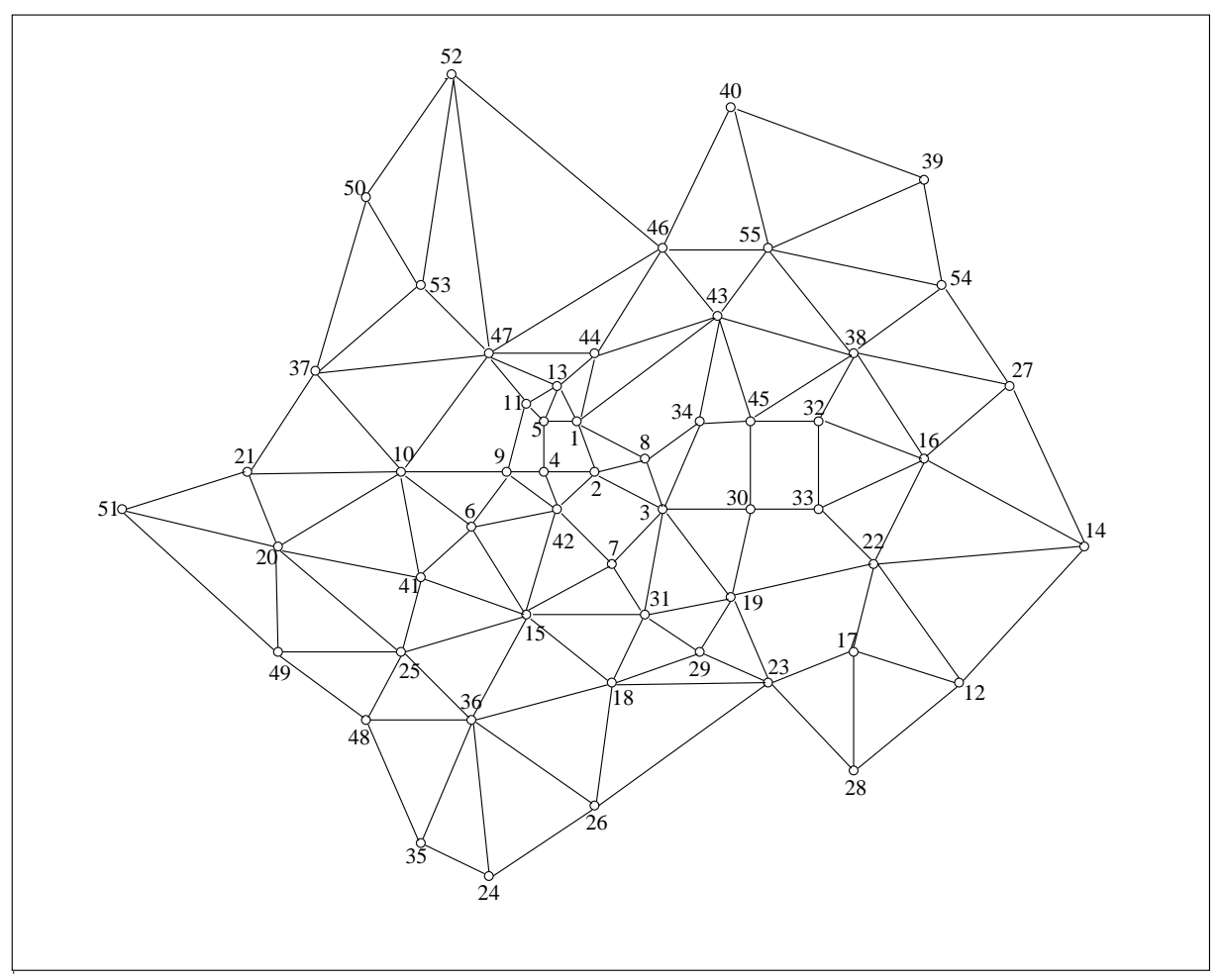


TABLE 1: Comparisons between the Heuristic and Enumeration algorithms

\begin{tabular}{|ccc|c|c|c|c|c|}
\hline$n$ & $\beta$ & $(p, q)$ & $\begin{array}{c}\text { NON } \\
\text { OPT }\end{array}$ & $\begin{array}{c}\text { AVGE } \\
\text { DEV }\end{array}$ & $\begin{array}{c}\text { MAX } \\
\text { DEV }\end{array}$ & $\begin{array}{c}\text { AVGE } \\
\text { CAPT }\end{array}$ & $\begin{array}{c}\text { AVGE } \\
\text { TIME }\end{array}$ \\
\hline 20 & 0.3 & $(2,2)$ & 0 & 0 & 0 & $53.4 \%$ & 6.0 \\
& & $(3,3)$ & 0 & 0 & 0 & $55.1 \%$ & 11.3 \\
& & $(4,4)$ & 1 & $5.2 \%$ & $5.2 \%$ & $54.5 \%$ & 16.6 \\
\hline 20 & 0.5 & $(2,2)$ & 0 & 0 & 0 & $59.2 \%$ & 5.8 \\
& & $(3,3)$ & 0 & 0 & 0 & $59.6 \%$ & 11.4 \\
& & $(4,4)$ & 1 & $1.9 \%$ & $1.9 \%$ & $57.1 \%$ & 17.1 \\
\hline 20 & 0.7 & $(2,2)$ & 0 & 0 & 0 & $66.1 \%$ & 6.4 \\
& & $(3,3)$ & 0 & 0 & 0 & $59.4 \%$ & 11.9 \\
& & $(4,4)$ & 1 & $3.2 \%$ & $3.2 \%$ & $50.4 \%$ & 17.0 \\
\hline 35 & 0.3 & $(2,2)$ & 0 & 0 & 0 & $60.1 \%$ & 9.4 \\
& & $(3,3)$ & 0 & 0 & 0 & $58.1 \%$ & 28.0 \\
& & $(4,4)$ & 0 & 0 & 0 & $56.9 \%$ & 37.3 \\
\hline 35 & 0.5 & $(2,2)$ & 0 & 0 & 0 & $64.6 \%$ & 15.5 \\
& & $(3,3)$ & 0 & 0 & 0 & $61.4 \%$ & 26.5 \\
& & $(4,4)$ & 1 & $1.2 \%$ & $1.2 \%$ & $61.0 \%$ & 40.3 \\
\hline 35 & 0.7 & $(2,2)$ & 0 & 0 & 0 & $67.9 \%$ & 15.5 \\
& & $(3,3)$ & 0 & 0 & 0 & $65.1 \%$ & 30.7 \\
& & $(4,4)$ & 1 & $8.1 \%$ & $8.1 \%$ & $67.0 \%$ & 33.7 \\
\hline 50 & 0.3 & $(2,2)$ & 0 & 0 & 0 & $57.5 \%$ & 16.8 \\
& & $(3,3)$ & 0 & 0 & 0 & $63.2 \%$ & 41.4 \\
& & $(4,4)$ & 0 & 0 & 0 & $58.4 \%$ & 70.2 \\
\hline \hline 50 & 0.5 & $(2,2)$ & 0 & 0 & 0 & $57.5 \%$ & 17.2 \\
& & $(3,3)$ & 0 & 0 & 0 & $60.6 \%$ & 40.1 \\
& & $(4,4)$ & 0 & 0 & 0 & $62.8 \%$ & 65.2 \\
\hline 50 & 0.7 & $(2,2)$ & 0 & 0 & 0 & $68.1 \%$ & 18.4 \\
& & $(3,3)$ & 2 & $8.2 \%$ & $14.1 \%$ & $69.3 \%$ & 34.4 \\
& & $(4,4)$ & 3 & $2.9 \%$ & $3.1 \%$ & $67.7 \%$ & 60.3 \\
\hline
\end{tabular}


TABLE 2: Results, 55-node network. Set of Rules 1

\begin{tabular}{|c|cccc|cc|}
\hline \multicolumn{3}{|c|}{} & & & \multicolumn{2}{c|}{$\begin{array}{c}\text { Total } \\
\text { Capture }\end{array}$} \\
\hline $\begin{array}{c}\text { Firm A Locations } \\
\text { Outlet Demand }\end{array}$ & 985 & 418 & 456 & 376 & 1735 & $48.5 \%$ \\
\hline $\begin{array}{c}\text { Firm B Locations } \\
\text { Outlet Demand }\end{array}$ & 965 & 351 & 276 & 248 & 1840 & $51.5 \%$ \\
\hline \hline Firm A Locations & 9 & 31 & 33 & 49 & & \\
Outlet Demand & 528 & 501 & 456 & 498 & 1983 & $55.5 \%$ \\
\hline Firm B Locations & 1 & 16 & 29 & 41 & & \\
Outlet Demand & 965 & 351 & 276 & closed & 1592 & $44.5 \%$ \\
\hline \hline Firm A Locations & 9 & 31 & 33 & 49 & & \\
Outlet Demand & 528 & 777 & 456 & 498 & 2259 & $63.2 \%$ \\
\hline Firm B Locations & 1 & 16 & 29 & 41 & & \\
Outlet Demand & 965 & 351 & closed & closed & 1316 & $36.8 \%$ \\
\hline \hline Firm A Locations & 9 & 31 & 33 & 49 & & \\
Outlet Demand & 528 & 777 & 807 & 498 & 2610 & $73.0 \%$ \\
\hline Firm B Locations & 1 & 16 & 29 & 41 & & \\
Outlet Demand & 965 & closed & closed & closed & 965 & $27.0 \%$ \\
\hline
\end{tabular}


TABLE 3: Results, Set of rules 2 vs. Set of Rules 1

\begin{tabular}{|c|c|c|c|c|}
\hline \multirow{2}{*}{$\begin{array}{c}\text { Initial } \\
p, q\end{array}$} & & \multicolumn{2}{|c|}{ Final } & $\begin{array}{c}\text { \% increase in } \\
\text { capture } \\
\text { with set of rules } 2\end{array}$ \\
& $p$ & $q$ & \\
2 & .60 & 2 & 2 & $2.3 \%$ \\
2 & .60 & 2 & 2 & $21.3 \%$ \\
3 & .60 & 3 & 3 & $0.8 \%$ \\
3 & .60 & 3 & 2 & $2.2 \%$ \\
3 & .40 & 3 & 2 & $8.5 \%$ \\
4 & .50 & 4 & 4 & $3.9 \%$ \\
4 & .50 & 4 & 3 & $8.8 \%$ \\
4 & .50 & 4 & 3 & $4.5 \%$ \\
4 & .40 & 4 & 3 & $2.9 \%$ \\
4 & .50 & 4 & 4 & $3.4 \%$ \\
\hline
\end{tabular}


TABLE 4: Results, 55-node network. Set of Rules 2

\begin{tabular}{|c|cccc|cc|}
\hline \multicolumn{2}{|c}{} & & & & \multicolumn{2}{c|}{$\begin{array}{c}\text { Total } \\
\text { Capture }\end{array}$} \\
\hline Firm A Locations & 2 & 13 & 23 & 32 & & \\
Outlet Demand & 714 & 415 & 371 & 295 & 1795 & $50.2 \%$ \\
\hline Firm B Locations & 1 & 16 & 29 & 41 & & \\
Outlet Demand & 266 & 305 & 241 & 868 & 1780 & $49.8 \%$ \\
\hline \hline Firm A Locations & 2 & 13 & 23 & 32 & & \\
Outlet Demand & 714 & 640 & 371 & 336 & 2061 & $57.6 \%$ \\
\hline Firm B Locations & 1 & 16 & 29 & 41 & & \\
Outlet Demand & closed & 305 & 341 & 868 & 1514 & $42.4 \%$ \\
\hline \hline Firm A Locations & 2 & 13 & 23 & 32 & & \\
Outlet Demand & 714 & 640 & 412 & 600 & 2366 & $66.2 \%$ \\
\hline Firm B Locations & 1 & 16 & 29 & 41 & & \\
Outlet Demand & closed & closed & 341 & 868 & 1209 & $33.8 \%$ \\
\hline \hline Firm A Locations & 2 & 13 & 23 & 32 & & \\
Outlet Demand & 714 & 640 & 692 & 661 & 2707 & $75.7 \%$ \\
\hline Firm B Locations & 1 & 16 & 29 & 41 & & \\
Outlet Demand & closed & closed & closed & 868 & 868 & $24.3 \%$ \\
\hline
\end{tabular}

